\title{
Effect of Neuritic Cables on Conductance Estimates for Remote Electrical Synapses
}

Astrid A. Prinz and Peter Fromherz

J Neurophysiol 89:2215-2224, 2003. First published 18 December 2002; doi:10.1152/jn.00956.2002

You might find this additional info useful...

This article cites 40 articles, 21 of which can be accessed free at:

/content/89/4/2215.full.html\#ref-list-1

This article has been cited by 1 other HighWire hosted articles

Membrane Capacitance Measurements Revisited: Dependence of Capacitance Value on

Measurement Method in Nonisopotential Neurons

Jorge Golowasch, Gladis Thomas, Adam L. Taylor, Arif Patel, Arlene Pineda, Christopher

Khalil and Farzan Nadim

J Neurophysiol, October , 2009; 102 (4): 2161-2175.

[Abstract] [Full Text] [PDF]

Updated information and services including high resolution figures, can be found at:

/content/89/4/2215.full.html

Additional material and information about Journal of Neurophysiology can be found at:

http://www.the-aps.org/publications/jn

This information is current as of January 19, 2015. 


\title{
Effect of Neuritic Cables on Conductance Estimates for Remote Electrical
}

\section{Synapses}

\author{
Astrid A. Prinz and Peter Fromherz \\ Department of Membrane and Neurophysics, Max Planck Institute for Biochemistry, D 82152 Martinsried, Germany
}

Submitted 24 October 2002; accepted in final form 11 December 2002

Prinz, Astrid A. and Peter Fromherz. Effect of neuritic cables on conductance estimates for remote electrical synapses. J Neurophysiol 89: 2215-2224, 2003. First published December 18, 2002; 10.1152/jn.00956.2002. The conductance of electrical synapses is usually estimated from voltage recordings at the neuronal somata under the assumption that each cell is isopotential. This approach neglects effects of intervening neurites. For a cell pair with unbranched neurites and an electrical synapse at their ends, we used cable theory to derive an analytical expression that relates the synaptic conductance to voltage recordings at the cell bodies and to the neurite properties. The equation implies that the conventional method significantly underestimates the actual synapse conductance if the neurite length is comparable to the electrotonic length constant and if the synaptic conductance is similar to the serial neurite conductance. For an experimental test, we cultured pairs of snail neurons on protein patterns, resulting in a geometry that matched the theoretical model. Using the isopotential theory, we estimated the synapse conductances and found them to be rather weak. To obtain the cable properties, we recorded spatiotemporal maps of signal propagation in the neurites using a voltage-sensitive dye. Fits of these maps to a passive cable model showed that the snail neurons are electrotonically rather compact. Given these features of our experimental system, the synaptic conductances derived with the nonisopotential model deviated from the estimates of the isopotential theory by about $13 \%$. This discrepancy, although small, shows that even in electrotonically compact neurons coupled by weak synapses the impact of the neuritic cables on conductance estimates cannot be neglected. When applied to less compact and more strongly coupled cell pairs in vivo, our approach can supply the realistic estimates of synaptic conductances that are necessary for a better understanding of the role of electrical coupling in neural systems.

\section{IN T R O D U C T I O N}

Electrical coupling occurs in many vertebrate and invertebrate neural circuits (Bennett 1997; Kiehn and Tresch 2002). Experimental and modeling studies suggest that electrical synapses can shape the output of neural networks in ways that go beyond their classical role in synchronization of neural systems and rapid signal transmission (Marder 1998) and allow them to perform such complex tasks as temporally precise coincidence detection (Edwards et al. 1998). In several systems where electrical synapses contribute to complex functions, the junctional conductance of the synapses has been identified as an important parameter for the performance of the circuit. An example for this is found in the mammalian inferior olive,

Address for reprint requests: P. Fromherz, Dept. of Membrane and Neurophysics, Max Planck Institute for Biochemistry, Am Klopferspitz 18a, D 82152 Martinsried, Germany (E-mail: fromherz@biochem.mpg.de). where electrical synapses--if they fall in the appropriate conductance range - can theoretically contribute to the rhythmogenesis in a population of not intrinsically oscillating neurons and where the strength of the electrical connections influences the period and amplitude of the emerging rhythm (Manor et al. 1997). Similarly, gap junctions between interneuron dendrites in the hippocampus can enhance the synchrony of gamma oscillations in spatially extended interneuron networks, but their ability to do so strongly depends on the junctional conductance of the electrical synapses (Traub et al. 2001). Another example are models of electrically coupled neuron pairs where the phase relationship between coupled oscillators, the cycle period and transitions between spiking and bursting behavior all depend on the junctional conductance (Sherman and Rinzel 1992). Knowledge of the conductances of electrical synapses in a neural circuit can therefore be crucial for the understanding of circuit function.

The common method of computing the conductance of a synapse from dual voltage recordings at the two cell bodies is based on the assumption of isopotential neurons (Bennett 1966) as illustrated in Fig. 1A. In intact circuits, however, the coupling site can be electrotonically remote from the sites of current injection and recording at the cell bodies of the coupled neurons. In such cases, the approach will necessarily underestimate the actual synaptic conductance by an amount that depends on the geometry and electrical properties of the neurites of both cells between the cell bodies and the coupling site.

In this paper, we address the problem of electrical coupling in nonisopotential neurons choosing a simple geometry of the neurons with an electrical synapse located at the tips of two unbranched neurites. Using cable theory as illustrated in Fig. $1 B$, we derive an analytical expression that relates the synaptic conductance to the passive cable properties and somatic recordings and to the apparent conductance given by the isopotential theory. The equation implies that conductance estimates obtained with the conventional method significantly differ from the local junctional conductance if the length of the two neurites is comparable to the electrotonic length constant and if their resistance is comparable to the resistance of the synapse.

For an experimental test, we used neurons from the snail Lymnaea stagnalis in culture. Their outgrowth in vitro can be guided by chemical patterns to achieve straight and unbranched neuritic cables. When two neurons grow on the same lane, their colliding growth cones readily formed nonrectifying electrical

The costs of publication of this article were defrayed in part by the payment of page charges. The article must therefore be hereby marked "advertisement" in accordance with 18 U.S.C. Section 1734 solely to indicate this fact. 


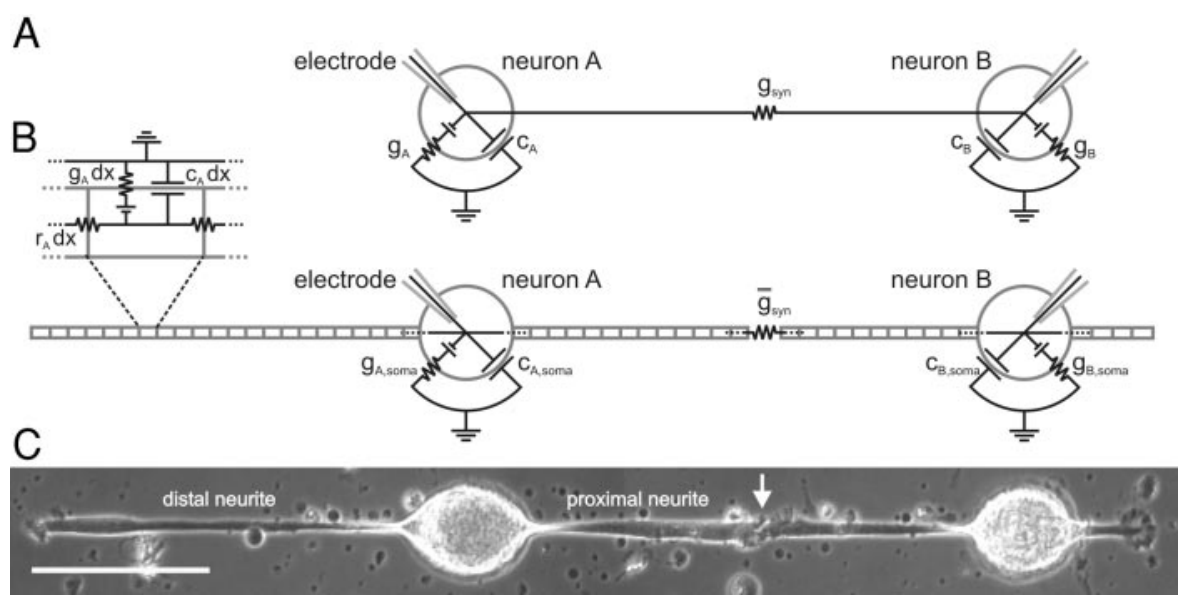

FIG. 1. Neuron pair with electrical synapse. A: conventional model for electrically coupled pair. The neurons are represented by single isopotential compartments with resting potentials $V_{\text {rest }}$, membrane capacitances $c$, ohmic input conductances $g$, and an effective synaptic conductance $g_{\text {syn }} B$ : equivalent circuit for nonisopotential neurons with unbranched neurites and a synaptic connection at the frontal contact of 2 neurites. Parameters are the membrane capacitances $c$, the membrane conductances $g$ and the cytoplasmic resistances $r$ per unit length of the neurites (differential length element $d x$ ), the synaptic conductance $\overline{\bar{g}}_{\text {syn }}$ and the capacitances $c_{\text {soma }}$ and conductances $g_{\text {soma }}$ of the somatic membranes. Battery symbols stand for resting potentials. $C$ : cultured pair of neurons from Lymnaea stagnalis with straight neurons on a lane of growth promoting factors. The white arrow indicates the position of the electrical synapse at the frontal contact of the 2 neurites. Scale bar: $100 \mu \mathrm{m}$. synapses (Prinz 2000; Prinz and Fromherz 2000) as shown in Fig. $1 C$. The technique allows the design of pairs of neurons that share a single point of contact with an electrical synapse at the terminal of two straight neurites - a geometry that corresponds to the one considered in our theoretical approach. Isolating the neurons from their surrounding tissue and restricting their neuritic tree to a straight cable has the additional advantage of facilitating the computation of the neuritic cable properties from high-resolution optical-imaging data obtained with voltage-sensitive dyes (Fromherz and Müller 1994). This alternative way of obtaining cable properties uses voltage transients observed throughout the neuritic cable rather than transients measured at the cell body alone. The imaging technique thus avoids the ill-posed parameter identification problem (White et al. 1992) associated with applications of the somatic shunt cable model to somatic voltage and current transients (Durand 1984; Kawato 1984).

\section{METHOD S}

\section{Substrate for guided growth}

Linearly patterned substrates for neurite guidance were prepared as previously described by UV lithography of a layer of brain-derived growth-promoting factors (Prinz and Fromherz 2000). Briefly, glass coverslips with attached silicone chambers (flexiPERM, In Vitro Systems and Services, Osterode, Germany) were incubated overnight with $1 \mathrm{mg} / \mathrm{ml}$ poly-L-lysine (Sigma, P6516) in Tris buffer $(0.15 \mathrm{M}, \mathrm{pH}$ 8.4) (Wong et al. 1983). After several washes with water, the chambers were filled with defined medium (PAN Systems, Aidenbach, Germany) containing (in $\mathrm{mM}$ ) $40.0 \mathrm{NaCl}, 1.7 \mathrm{KCl}, 4.1 \mathrm{CaCl}_{2}, 1.5$ $\mathrm{MgCl}_{2}, 1.0$ glutamine, and 10.0 HEPES ( $\mathrm{pH}$ 7.9), all other ingredients of Leibovitz L-15 medium at half of the standard concentration, and $20 \mu \mathrm{g} / \mathrm{ml}$ gentamycin (Sigma, G3632) (Ridgway et al. 1991). Central ganglionic rings were isolated from $L$. stagnalis that were raised in tap water and fed on lettuce. Prior to dissection, the snails were deshelled and soaked in $25 \%$ Listerine in normal saline consisting of (in $\mathrm{mM}$ ) $51.3 \mathrm{NaCl}, 1.7 \mathrm{KCl}, 4.1 \mathrm{CaCl}_{2}, 1.5 \mathrm{MgCl}_{2}$, and 5.0 HEPES 5.0 at $\mathrm{pH}$ 7.9 (all from Sigma), then pinned to silicone elastomer (Sylgard) in antibiotic saline, which is normal saline with $150 \mu \mathrm{g} / \mathrm{ml}$ gentamycin (Ridgway et al. 1991). The dissected brains were extensively washed in antibiotic saline and added to the defined medium in the chambers at 3 brains $/ \mathrm{ml}$. After $3-5$ days at $20^{\circ} \mathrm{C}$, the conditioned supernatant medium and the brains were removed. The cover slips were dried and illuminated for $20 \mathrm{~min}$ with the full spectrum of a mercury lamp (Osram HBO200) through a mask consisting of aluminum strips (14 $\mu \mathrm{m}$ wide and $33 \mu \mathrm{m}$ apart) on a silica plate (Fromherz and Schaden
1994). Then the chamber was filled with defined medium or with defined medium mixed with the conditioned medium at a ratio of $1: 1$.

\section{Cell culture}

Neurons were isolated from the A-clusters in the pedal ganglia of $L$. stagnalis and cultured following standard procedures (Ridgway et al. 1991) as follows: after removing the outer connective tissue, snail brains were incubated in antibiotic saline for $15 \mathrm{~min}$ and in defined medium with $1.33 \mathrm{mg} / \mathrm{ml}$ collagenase/dispase (Boehringer Mannheim) and $0.67 \mathrm{mg} / \mathrm{ml}$ trypsin (Sigma, T8253) for 30-35 min, washed several times with defined medium, treated with $0.67 \mathrm{mg} / \mathrm{ml}$ trypsin inhibitor (Sigma, T9003) in defined medium for $15 \mathrm{~min}$, and again washed several times. Then a high-osmolarity medium was applied, consisting of defined medium with $30 \mathrm{mM}$ glucose added. The pedal ganglia were opened using a micro-needle. Neurons from the A-clusters were removed by aspiration through a fire-polished and siliconized (Sigmacote, Sigma) micropipette, immediately placed on the patterned substrate, and incubated for 1 day at $20^{\circ} \mathrm{C}$ (Ridgway et al. 1991). The cells were positioned in pairs at distances of several hundred $\mu \mathrm{m}$ on the same lane for the formation of synapses or positioned in isolation for the imaging experiments.

\section{Electrophysiology}

Both for the cell pair experiments and for the optical imaging experiments, neurons were impaled with sharp microelectrodes pulled from glass capillaries, filled with a saturated solution of $\mathrm{K}_{2} \mathrm{SO}_{4}$ and contacted with a chlorinated silver wire. Electrode resistances were $10-25 \mathrm{M} \Omega$. An electrode amplifier operating in discontinuous mode (Ba-1S, npi Advanced Electronic Systems, Germany) was used to inject current and monitor voltage. We applied hyperpolarizing currents of up to $0.5 \mathrm{nA}$ amplitude and $900 \mathrm{~ms}$ duration in the experiments with cell pairs and hyperpolarizing current pulses of 10-ms duration and up to $2 \mathrm{nA}$ amplitude in the imaging experiments.

\section{Optical recording}

The voltage-sensitive dye dibutyl-naphtalene-butylsulfonato-isoquinolinium (BNBIQ) (Ephardt and Fromherz 1993; Fromherz and Müller 1993) was dissolved at a concentration of $4.3 \mathrm{mM}$ in $15 \mathrm{mM}$ cholic acid (Sigma) (Meyer et al. 1997) in Lymnaea cell culture medium (Ridgway et al. 1991). To stain cultured snail neurons, this stock solution was diluted 50-fold with medium and immediately added to the culture chamber, which was then incubated for $10 \mathrm{~min}$ in the dark and subsequently washed with medium. To achieve satisfactory staining of the neural membrane, the staining procedure was repeated up to 10 times.

The straight and unbranched neurites of the stained cell were 
projected onto a linear array of 100 photodiodes (Müller 1994) (LD50-5, Centronic, England) through an inverted microscope (Axiovert 135, Zeiss, Germany), and the cell body was impaled with microelectrodes. For dye excitation, the light from a Xenon lamp was passed through a 450 to $190 \mathrm{~nm}$ band-pass onto the cell. The fluorescent light emitted by the dye in the somatic and neuritic membrane was long-pass filtered at $560 \mathrm{~nm}$ and recorded at a spatial resolution of 4-5 $\mathrm{m}$ as previously described (Fromherz and Müller 1994; Meyer et al. 1997) while hyperpolarizing current pulses were injected into the cell.

When illuminated, the voltage-sensitive dye bleaches and the fluorescence intensity therefore decays over the exposure time. To separate this effect from the fluorescence change due to neuronal activity, we corrected the recorded fluorescence transient for each diode. This was achieved by fitting the parts of the fluorescence transient during which the cell was at resting potential to a function of the form $f(t)=$ $a+b^{*} e^{-t / c}$ and subtracting this baseline from the recorded transient (Fromherz and Vetter 1992). The difference to baseline was normalized to an amplitude of -1 for each diode individually to account for the unknown proportionality factor between the local membrane potential change and the recorded fluorescence change. The normalized transients obtained in this manner thus contain information about shape and timing of the local voltage transient but not about its amplitude.

\section{Estimation of cable properties from imaging data}

To estimate the electrical properties of cultured snail neurons with straight neurites, we used a model consisting of homogeneous cylindrical cables joined at one end to a spherical soma and sealed at the other end. The length $l$ and diameter $d$ of each cable were matched to the dimensions of the grown neurite. The resistance per unit length $r$ is related to the cytoplasmic resistivity $R$ as $r=4 R /\left(d^{2} \pi\right)$, the membrane conductance per unit length $g$ to the membrane conductance $G$ per unit area as $g=G d \pi$ and the capacitance per unit length $c$ to the membrane capacitance $C$ per unit area as $c=C d \pi$. The total capacitance $c_{\text {soma }}$ and conductance $g_{\text {soma }}$ of the cell body depend on the diameter $d_{\text {soma }}$ and the capacitance per unit area $C_{\text {soma }}$ and conductance per unit area $G_{\text {soma }}$ as $c_{\text {soma }}=C_{\text {soma }} d_{\text {soma }}^{2} \pi$ and $g_{\text {soma }}=$ $G_{\text {soma }} d_{\text {soma }}^{2} \pi$. The model neuron was stimulated by injecting a hyperpolarizing current pulse into the soma. The dynamics of the membrane potential at the soma and along the neurites were obtained by numerical solution of the cable equation using an Euler forward algorithm (Press et al. 1997).

The voltage transient in each compartment of the model neuron in response to the hyperpolarizing somatic current pulse was normalized to an amplitude of -1 . This was necessary to allow a comparison of the simulated voltage transients and the recorded fluorescence transients, which were normalized due to the lack of amplitude information inherent to the imaging technique. Thus the fit relied solely on the delay and shape of voltage transients at different locations along the neurites. A fit of only the timing and shape of the transients is sufficient because both of these features are influenced by the cable parameters and therefore contain information about them.

We adjusted the electrical parameters $r, g$, and $c$ of the model neurites and the parameters $g_{\text {soma }}$ and $c_{\text {soma }}$ of the model cell body to achieve an optimal fit between the normalized voltage transients in the model and the normalized optical records. To reduce the number of parameters, we assigned the standard capacitance $C=1 \mu \mathrm{F} / \mathrm{cm}^{2}$ to the neurite membrane. An initial estimate of the somatic membrane conductance $G_{\text {soma }}$ was obtained from the experimental input conductance $\overline{\bar{g}}$ of a soma with sealed neurites. We used the relation $\overline{\bar{g}} \approx$ $G_{\text {soma }} \pi\left(d_{\text {soma }}^{2}+\Sigma d l\right)$ with length $l$ and diameter $d$ of the neurites, implying that the neuron is electrotonically compact and that the specific membrane conductances of cell body and neurites are similar. For given diameter $d_{\text {soma }}$ of the soma and known diameters $d$ and lengths $l$ of the neurites, we chose a value for $C_{\text {soma }}$ and varied $G$ and
$R$ to minimize the deviation of the computed normalized voltage transients from the normalized experimental fluorescence transients. Let $S_{n d}^{\exp }$ and $S_{n d}^{\mathrm{sim}}$ be the experimental and simulated normalized signals for data point $d$ of diode $n$, and let $\sigma_{n}$ be the SD of the transient of diode $n$ prior to the onset of current injection. With $N$ fluorescence transients and $D$ data points per transient, we used $\chi^{2} /(N D)$ as a measure for the deviation where $\chi^{2}=\sum_{n d}\left[\left(S_{n d}^{\exp }-S_{n d}^{\text {sim }}\right) / \sigma_{n}\right]^{2}$. The fit procedure was repeated for different values of $C_{\text {soma }}$ to find a set $C_{\text {soma }}, G$, and $R$ that best reproduced the recorded somatic voltage transient and the normalized optical recordings along the neurites. Because the somatic membrane conductance $G_{\text {soma }}$ used as an input parameter for this fit was only a rough estimate (see preceding text), we repeated the fit procedure with other values of $G_{\text {soma }}$ in a physiologically meaningful range to evaluate to what extent the fit results for $C_{\text {soma }}, G$, and $R$ depend on the particular choice of $G_{\text {soma. }}$.

\section{Synaptic coupling coefficient}

The strength of an electrical synapse is usually described in terms of the stationary voltage changes in the postsynaptic and presynaptic cell caused by a hyperpolarizing current injection into the presynaptic neuron. Given the stationary voltages without (denoted by 0 ) and with (denoted by $\infty$ ) an injection current $i_{\text {inj, }}$, the coupling coefficient $k_{\text {pre-post }}$ is defined by Eq. 1 with the voltage changes $\Delta V_{\text {pre }}=V_{\text {pre }}^{\infty}-$ $V_{\text {pre }}^{0}$ and $\Delta V_{\text {post }}=V_{\text {post }}^{\infty}-V_{\text {post }}^{\mathrm{o}}($ Bennett 1966)

$$
k_{\text {pre-post }}=\frac{\Delta V_{\text {post }}}{\Delta V_{\text {pre }}}
$$

\section{Synaptic conductance assuming isopotential neurons}

The conventional model to describe two electrically coupled passive neurons is shown in Fig. 1A (Bennett 1966), although Bennett implicitly assumed the special case of $V_{\text {pre,rest }}=V_{\text {post,rest }}$. The parameters are an effective synaptic conductance $g_{\text {syn }}$, the input conductances $g_{\text {pre }}$ and $g_{\text {post }}$ of the pre- and postsynaptic neuron and the resting potentials $V_{\text {pre,rest }}$ and $V_{\text {post,rest }}$. An injection current $i_{\text {inj }}$ in the presynaptic cell gives rise to a change of the stationary currents through the presynaptic membrane, the synapse and the postsynaptic membrane. Equations 2 and 3 are the steady-state current balances for both neurons

$$
\begin{aligned}
& g_{\text {pre }}\left(V_{\text {pre }}-V_{\text {pre,rest }}\right)+g_{\text {syn }}\left(V_{\text {pre }}-V_{\text {post }}\right)=i_{\text {inj }} \\
& g_{\text {post }}\left(V_{\text {post }}-V_{\text {post,rest }}\right)+g_{\text {syn }}\left(V_{\text {post }}-V_{\text {pre }}\right)=0
\end{aligned}
$$

We apply these relations to the steady states before and after a current step from 0 to $i_{\text {inj }}$ in the presynaptic cell. When we subtract the two versions of Eqs. 2 and 3 before and after the step from each other, we obtain Eqs. 4 and 5, where $\Delta V_{\text {pre }}$ and $\Delta V_{\text {post }}$ are the changes in the steady-state voltages induced by the current step

$$
\begin{aligned}
& g_{\text {pre }} \Delta V_{\text {pre }}+g_{\text {syn }}\left(\Delta V_{\text {pre }}-\Delta V_{\text {post }}\right)=i_{\text {inj }} \\
& g_{\text {post }} \Delta V_{\text {post }}+g_{\text {syn }}\left(\Delta V_{\text {post }}-\Delta V_{\text {pre }}\right)=0
\end{aligned}
$$

From Eq. 5 follows Eq. 6 for the ratio of the post- and the presynaptic voltage change, which corresponds to the coupling coefficient $k_{\text {pre-post }}$ according to Eq. 1

$$
\frac{\Delta V_{\text {post }}}{\Delta V_{\text {pre }}}=\frac{g_{\text {syn }}}{g_{\text {syn }}+g_{\text {post }}}
$$

Equation 6 shows that the coupling coefficient does not reflect the synaptic conductance $g_{\text {syn }}$ alone, but that it depends on the input conductance $g_{\text {post }}$ of the postsynaptic neuron (Bennett 1966).

Using $E q$. 4, we eliminate the presynaptic voltage change $\Delta V_{\text {pre }}$ from $E q .6$ and solve for $\Delta V_{\text {post }}$ to obtain $E q .7$ 


$$
\Delta V_{\text {post }}=\frac{g_{\text {syn }}}{\left(g_{\text {pre }}+g_{\text {post }}\right) g_{\text {syn }}+g_{\text {pre }} g_{\text {post }}} i_{\text {inj }}
$$

According to $E q .7$, the postsynaptic response $\Delta V_{\text {post }}$ is symmetric in the input conductances $g_{\text {pre }}$ and $g_{\text {post }}$ of the two neurons. This means that the postsynaptic voltage change is the same no matter which of the two neurons is postsynaptic.

Finally, we can express the synaptic conductance $g_{\text {syn }}$ in terms of experimentally accessible voltage changes $\Delta V_{\text {pre }}$ and $\Delta V_{\text {post }}$. To do so, we first stimulate neuron $A$ in a cell pair with a current step from 0 to $i_{\text {inj }}$ (such that $\mathrm{A}$ is pre and $\mathrm{B}$ is post), and then apply the same current step to neuron B (such that B is pre and A is post). From the two versions of Eqs. 4 and 5 for the two directions of signal transfer we can derive $E q .8$

$$
g_{\text {syn }}=\frac{\Delta V_{\text {post }}}{\Delta V_{\text {pre,A }} \Delta V_{\text {pre }, \mathrm{B}}-\Delta V_{\text {post }, \mathrm{A}} \Delta V_{\text {post }, \mathrm{B}}} i_{\text {inj }}
$$

Here, $\Delta V_{\text {pre,A }}$ is the voltage change in neuron $\mathrm{A}$ when it is presynaptic (and analogously for $\Delta V_{\text {post, } \mathrm{A}}, \Delta V_{\text {pre, } \mathrm{B}}$, and $\Delta V_{\text {post,B }}$ and $\Delta V_{\text {post }}=$ $\Delta V_{\text {post, } \mathrm{A}}=\Delta V_{\text {post,B }}$ according to $E q$. 7 .

\section{Synaptic conductance assuming nonisopotential neurons}

Figure $1 B$ shows the circuit for two electrically coupled neurons that takes the passive cable properties of the neurites between the cell bodies and the synapse at their tips into account. In steady state, the current balances for the two neurons are given by Eqs. 9 and 10

$$
\begin{gathered}
\left(g_{\text {pre,soma }}+g_{\text {pre,dist }}\right)\left(V_{\text {pre }}-\overline{\bar{V}}_{\text {pre,est }}\right)+i_{\text {pre,prox }}^{\text {memb }}+\overline{\bar{g}}_{\text {syn }}\left(V_{\text {pre }}^{\text {tip }}-V_{\text {post }}^{\text {tip }}\right)=i_{\text {inj }} \\
\left(g_{\text {post,soma }}+g_{\text {post,dist }}\right)\left(V_{\text {post }}-\overline{\bar{V}}_{\text {post,rest }}\right)+i_{\text {post,prox }}^{\text {memb }}+\overline{\bar{g}}_{\text {syn }}\left(V_{\text {post }}^{\text {tip }}-V_{\text {pre }}^{\text {tip }}\right)=0
\end{gathered}
$$

In these equations, $g_{\text {pre,soma }}$ is the membrane conductance of the presynaptic soma, $g_{\text {pre,dist }}$ is the total input conductance of its distal neurite or neurites, $i$ memb is the membrane current of its proximal neurite, and $V_{\text {pre }}$ and $V_{\text {pre }}^{\text {tip }}$ are its membrane potential at the soma and at the tip of the proximal neurite, respectively. The presynaptic resting potential $\overline{\bar{V}}_{\text {pre,rest }}$ and the junctional conductance $\overline{\bar{g}}_{\text {syn }}$ of the synapse have cable-like overbars to distinguish them from the corresponding parameters in the isopotential model, and all postsynaptic parameters are named in complete analogy to the presynaptic ones.

Equations 9 and 10 contain the experimentally not accessible membrane currents $i_{\text {pre,prox }}^{\text {memb }}$ and $i_{\text {post,prox }}^{\text {memb }}$ and tip potentials $V_{\text {pre }}^{\text {tip }}$ and $V_{\text {post }}^{\text {tip }}$ that don't occur in the current balances Eqs. 2 and 3 of the isopotential model. To allow a comparison between the two models, we use cable theory to express these currents and potentials in terms of electrical properties of the neurons and somatic potentials (see APPENDIX). After these substitutions, the current balances can be brought into the same mathematical form as the balances in the isopotential model, resulting in the (slightly unwieldy) Eqs. 11 and 12

$$
\begin{gathered}
{\left[\overline{\bar{g}}_{\text {pre }}+\gamma(\chi-1)\right]\left[V_{\text {pre }}-\overline{\bar{V}}_{\text {pre,rest }}-\frac{\left(\cosh L_{\text {post }}-1\right)\left(\overline{\bar{V}}_{\text {post,rest }}-\overline{\bar{V}}_{\text {pre,rest }}\right)}{\frac{\bar{g}_{\text {pre }}}{\gamma}+\chi-1}\right]} \\
+\gamma\left(V_{\text {pre }}-V_{\text {post }}\right)=i_{\text {inj }} \\
{\left[\overline{\bar{g}}_{\text {post }}+\gamma\left(\frac{1}{\chi}-1\right)\right]\left[\begin{array}{c}
V_{\text {post }}-\overline{\bar{V}}_{\text {post, rest }}-\frac{\left(\cosh L_{\text {pre }}-1\right)\left(\overline{\bar{V}}_{\text {pre,rest }}-\overline{\bar{V}}_{\text {post,rest }}\right)}{\frac{\bar{g}_{\text {post }}}{\gamma}+\frac{1}{\chi}-1} \\
+\gamma\left(V_{\text {post }}-V_{\text {pre }}\right)=0
\end{array}\right.}
\end{gathered}
$$

Here

$\overline{\bar{g}}_{\text {pre }}=g_{\text {pre,soma }}+g_{\text {pre,dist }}+\frac{\tanh L_{\text {pre }}}{\lambda_{\text {pre }} r_{\text {pre }}} \quad$ and $\quad \overline{\bar{g}}_{\text {post }}=g_{\text {post,soma }}+g_{\text {post,dist }}+\frac{\tanh L_{\text {post }}}{\lambda_{\text {post }} r_{\text {post }}}$ stand for the input resistances that the two neurons would have if their proximal neurites were sealed. $L_{\text {pre }}$ and $L_{\text {post }}$ are the electrotonic lengths of the proximal neurites, $\lambda_{\text {pre }}$ and $\lambda_{\text {post }}$ are their electrotonic length constants, $r_{\text {pre }}$ and $r_{\text {post }}$ are their axial resistances per unit length, and we have introduced the abbreviations, Eqs. 14 and 15

$$
\begin{gathered}
\chi=\frac{\cosh L_{\text {post }}}{\cosh L_{\text {pre }}} \\
\frac{1}{\gamma}=\left(\frac{1}{\bar{g}_{\text {syn }}}+\lambda_{\text {pre }} r_{\text {pre }} \tanh L_{\text {pre }}+\lambda_{\text {post }} r_{\text {post }} \tanh L_{\text {post }}\right) \cosh L_{\text {pre }} \cosh L_{\text {post }}
\end{gathered}
$$

Comparison of the coeffients in the current balances Eqs. 11 and 12 with the balances Eqs. 2 and 3 in the isopotential model immediately suggests relations between the input conductances $g_{\text {pre }}$ and $g_{\text {post }}$, the resting potentials $V_{\text {pre,rest }}$ and $V_{\text {post,rest }}$ and the synaptic conductance $g_{\text {syn }}$ in the isopotential model and the corresponding parameters $\overline{\bar{g}}_{\text {pre }}$, $\overline{\bar{g}}_{\text {post }}, \overline{\bar{V}}_{\text {pre,rest }}, \overline{\bar{V}}_{\text {post,rest }}$, and $\overline{\bar{g}}_{\text {syn }}$ in the nonisopotential model. In particular, that comparison suggests the identification $g_{\text {syn }}=\gamma$.

The equation $1 g_{\text {syn }}=\gamma$ is confirmed by using the same transformations that led from the current balances Eqs. 2 and 3 to expression $E q .8$ in the isopotential model. When we apply these transformations to the current balances Eqs. 11 and 12 we obtain Eq. 16

$$
\gamma=\frac{\Delta V_{\text {post }}}{\Delta V_{\text {pre }, \mathrm{A}} \Delta V_{\text {pre, } \mathrm{B}}-\Delta V_{\text {post }, \mathrm{A}} \Delta V_{\text {post }, \mathrm{B}}} i_{\text {inj }}
$$

Comparison of Eqs. 16 and 8 shows that $g_{\text {syn }}$ and $\gamma$ are indeed identical. Together with the definition $(E q .15)$ of $\gamma$, we obtain the crucial relation Eq. 17 between the synaptic conductance $\overline{\bar{g}}_{\text {syn }}$ and the estimate $g_{\text {syn }}$ given by the isopotential model

$$
\frac{1}{\overline{\bar{g}}_{\text {syn }}}=\frac{1}{g_{\text {syn }}} \frac{1}{\cosh L_{\text {pre }} \cosh L_{\text {post }}}-\lambda_{\text {pre }} r_{\text {pre }} \tanh L_{\text {pre }}-\lambda_{\text {post }} r_{\text {post }} \tanh L_{\text {post }}
$$

A complete neglect of the neurites leads to $g_{\text {syn }}=\overline{\bar{g}}_{\text {syn }}$. For electrotonically short neurites with $L_{\text {pre }} \ll 1$ and $L_{\text {post }} \ll 1$, we obtain $E q$. 18 , a relation that reflects the serial resistances of the synapse and the proximal neurites

$$
\frac{1}{\overline{\bar{g}}_{\text {syn }}}=\frac{1}{g_{\text {syn }}}-r_{\text {pre }} l_{\text {pre }}-r_{\text {post }} l_{\text {post }}
$$

Assuming $r_{\text {pre }}=r_{\text {post }}=r$ with a total neurite length $l=l_{\text {pre }}+l_{\text {post }}$, we obtain the relation $\overline{\bar{g}}_{\text {syn }}=g_{\text {syn }} /\left(1-g_{\text {syn }} r l\right)$ for $g_{\text {syn }} r l<l$. In this parameter range, the ratio of the apparent conductance from the isopotential theory and the synaptic conductance from the cable theory drops for increasing neurite lengths, for increasing neurite resistances, and for increasing synaptic conductances.

\section{R E S U L T S}

We describe current-clamp experiments with neuron pairs from L. stagnalis joined by unbranched neurites with an electrical synapse at their tips. We first evaluate the experimental data in terms of the conventional theory that assumes isopotential neurons. Then, as a basis for the evaluation with the nonisopotential model, the cable parameters of the neurites are determined from optical mapping of voltage transients in the neurites. Using these results, we compute the junctional conductances of the electrical synapses and compare them to the estimates from the isopotential approach.

\section{Synaptic coupling coefficient of Lymnaea neuron pairs}

Neurons isolated from the A clusters in the pedal ganglia of pond snails were cultured on linearly patterned substrates as previously described (Prinz and Fromherz 2000). Over the 
course of one day in culture, most cells extended unbranched neurites along the straight lanes of growth-promoting substrate. When two cell bodies were positioned on the same lane at a distance of several hundred micrometers, their growth cones could make a contact as illustrated in Fig. 1C. Figure 2 shows recordings from such a pair of cultured neurons with simple neurite geometry (Fig. 2A). The voltage transients caused by depolarizing and hyperpolarizing current injections in either cell were accompanied by postsynaptic potential changes in the other cell (Fig. 2B). The transfer of hyperpolarization demonstrated that the coupling at the contact site was electrical. Figure $2 C$ shows families of hyperpolarizing injection currents of increasing amplitude and the pre- and postsynaptic voltage transients they caused.

From the pre- and postsynaptic steady-state voltages in each cell before and after the current step we computed the coupling coefficient $k_{\text {pre-post }}$ of the electrical synapse according to $E q$. 1 . We found no voltage dependence of the coupling between these cultured neurons (Prinz 2000), so we averaged the coupling coefficients obtained with different injection currents for each cell pair and each direction of signal transfer. From the data shown in Fig. $2 C$ we obtained $k_{\text {pre-post }}=0.18$ from cell A to cell $\mathrm{B}$ and $k_{\text {pre-post }}=0.08$ from cell $\mathrm{B}$ to cell $\mathrm{A}$. The difference is due to different input conductances of both neurons according to $E q$. 6. Figure $3 A$ shows the cumulative distribution function of the coupling coefficients $k_{\text {pre-post }}$ of 11 cell pairs. We found a wide range of coupling strengths between extreme values $k_{\text {pre-post }}=0.01$ and $k_{\text {pre-post }}=0.27$ with an average of $k_{\text {pre-post }}=0.09$.

\section{Synaptic conductance under the assumption of isopotential cells}

To estimate the synaptic conductance $g_{\text {syn }}$ under the assumption of isopotential cells, we used relation $E q$. 8 for the 11 cell pairs considered in the preceding paragraph and again averaged the results for different injection currents in each cell pair. Figure $3 B$ shows the cumulative distribution function of $g_{\text {syn }}$. The synapse conductances are rather small, with an average of $g_{\text {syn }}=0.33 \mathrm{nS}$ and extreme values of $g_{\text {syn }}=0.04 \mathrm{nS}$ and $g_{\text {syn }}=0.98 \mathrm{nS}$.

In Fig. $3 C$, the coupling coefficients $k_{\text {pre-post }}$ are plotted versus the conductances $g_{\text {syn }}$ for the 11 neuron pairs. The figure shows that $k_{\text {pre-post }}$ is not well correlated to $g_{\text {syn }}$ and therefore conveys little information about the strength of the underlying synapse. The discrepancy must be assigned to the variability of the input conductance $g_{\text {post }}$ of the postsynaptic neurons that affects the coupling coefficient for isopotential neurons according to $E q$. 6 .

\section{Cable parameters from optical imaging}

To determine the cable parameters of the neurites, individual neurons from the A clusters were cultured on linearly patterned substrates, stained with the voltage-sensitive dye BNBIQ and impaled with a microelectrode. A brief hyperpolarizing current pulse was injected into the soma, and the change of fluorescence intensity along the neurites was recorded with a photodiode array and processed as described in METHODS.

The outline drawing of a neuron with two straight neurites is shown in Fig. 4A. The somatic voltage response to a $10 \mathrm{~ms}$

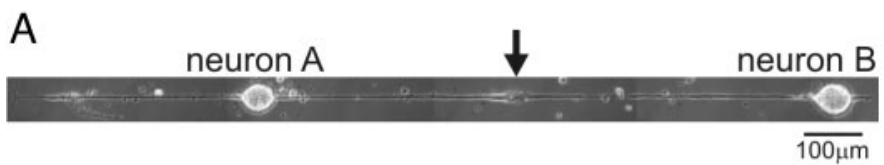

B
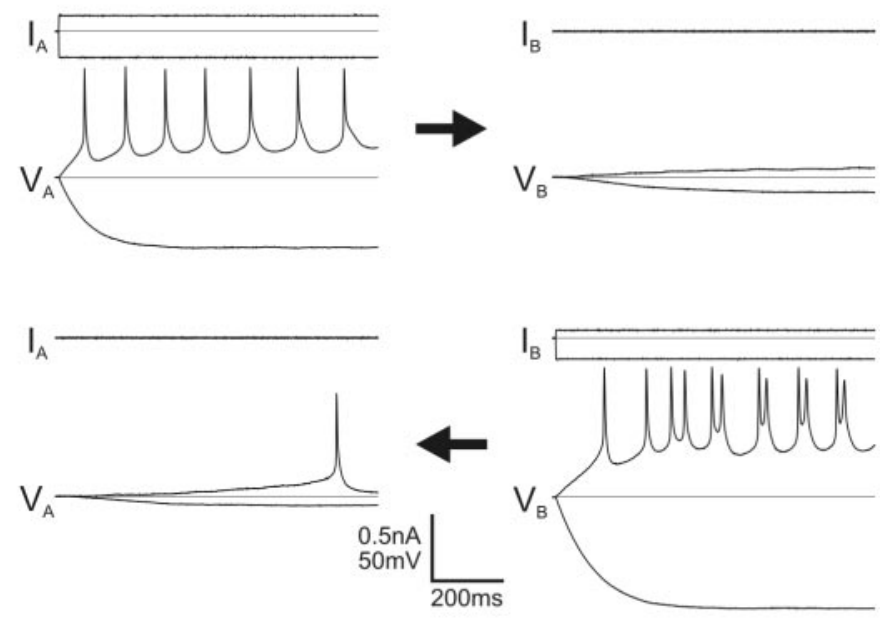

C

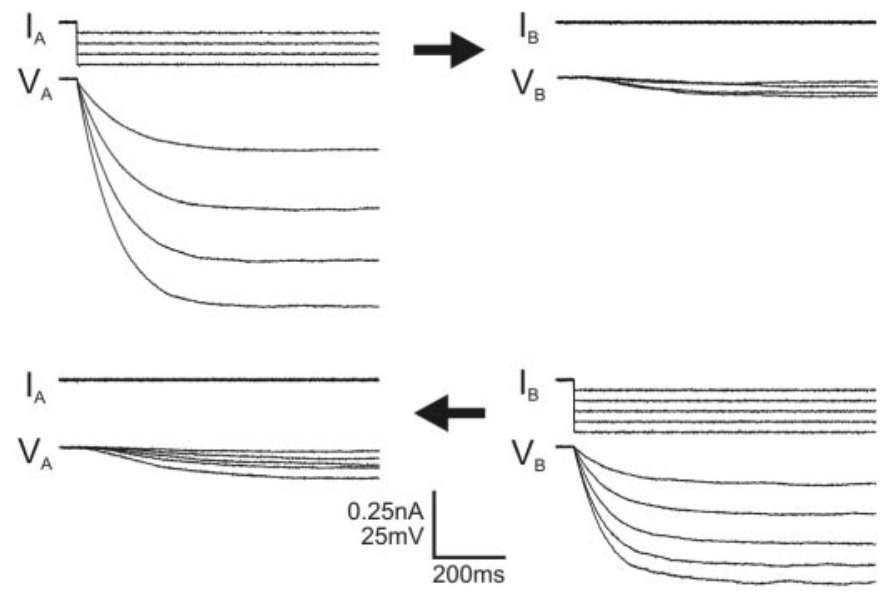

FIG. 2. Electrical coupling between pair of geometrically simple neurons. $A$ : micrograph of 2 Lymnaea neurons whose growth cones touch after 1 day of culture on a linearly patterned substrate. The arrow indicates the location of the electrical synapse between the 2 growth cones. Scale bar: $100 \mu \mathrm{m} . B$ : comparison of depolarizing and hyperpolarizing current steps injected into the cells shown in $A$. Current and voltage traces for both cells in response to injection into neuron A (top) and neuron B (bottom). The corresponding injection currents are shown above each set of voltage traces. Horizontal lines in each panel indicate 0 injection current and resting potential. Black arrows show the direction of signal transfer. Hyperpolarizing and depolarizing voltages changes can spread through the synapse in either direction. $C$ : comparison of different hyperpolarizing current steps. Current and voltage traces for current injected into cell A (top) and cell B (bottom). Injection currents are plotted above each set of voltage traces. Families of traces like these were used to estimate the conductance of the electrical synapse as described in the text.

current pulse is depicted in Fig. 4B. The spatiotemporal fluorescence pattern in the neurites is displayed as a color-coded map in Fig. 4C with the bleach-corrected fluorescence transients normalized to a peak value of -1 for each diode. Obviously, the hyperpolarization propagates rather fast through the entire cell without significant broadening. The neuron seems to 

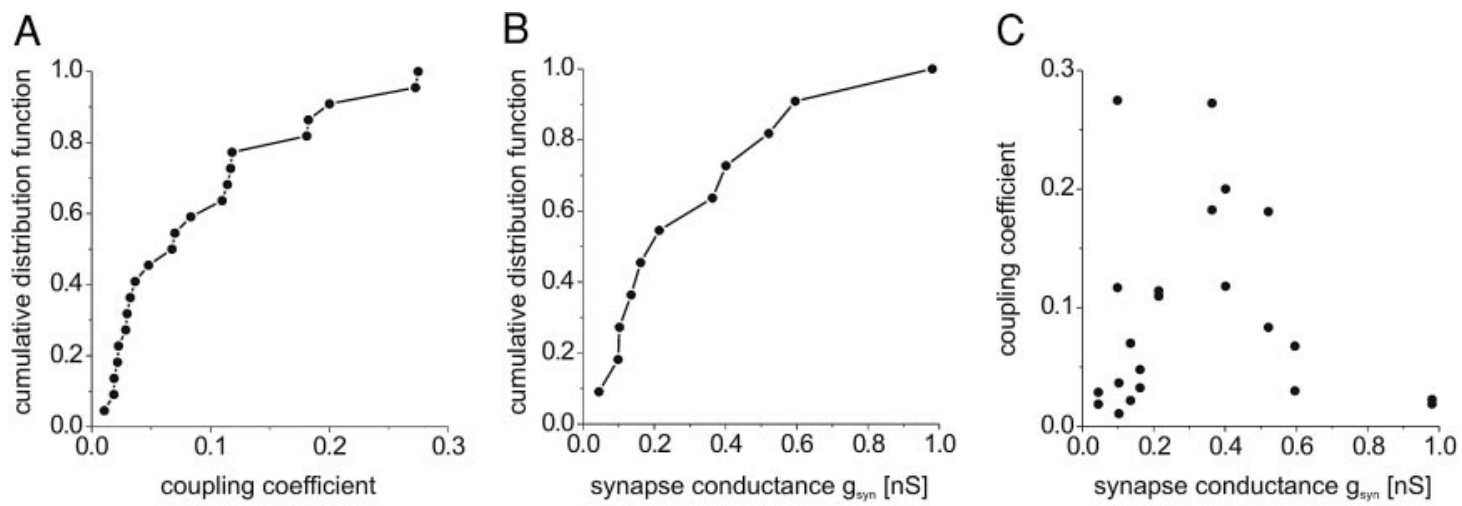

FIG. 3. Coupling coefficient and synaptic conductance with isopotential theory. A: cumulative distribution function of the coupling coefficients $k_{\text {pre-post }}$ obtained from current step injections in 11 geometrically simple pairs of snail neurons with a single point of contact. Note that there are 2 coupling coefficients for every cell pair -1 for each direction of signal transfer. $B$ : cumulative distribution function of synapse conductances $g_{\text {syn }}$ computed for the same cell pairs under the assumption of isopotential cells. $C$ : coupling coefficients $k_{\text {pre-post }}$ plotted vs. $g_{\text {syn }}$. The two measures of synapse strength are not well correlated.

be electrotonically fairly compact. Nonetheless, a careful inspection shows that the signal exhibits a delay along the neurite.

We determined the soma and neurite dimensions of the neuron from a photomicrograph of the cell and constructed a model neuron of the same dimensions; in the case of the neuron in Fig. 4, the soma diameter was $57 \mu \mathrm{m}$, the neurite lengths were 311 and $154 \mu \mathrm{m}$ and the neurite diameter was $7 \mu \mathrm{m}$, a diameter typical for Lymnaea neurites grown on patterned substrates. With this model neuron, we simulated voltage transients in response to the same hyperpolarizing current pulse used in the experiment and fitted them to the fluorescence transients as described in METHODS. The simulated somatic voltage transient was superimposed on the experimental transient in Fig. $4 B$, and the simulated spatiotemporal voltage in the neurite is displayed as a contour map in Fig. $4 C$. The electrical parameters obtained from the fit were $C_{\text {soma }}=1.5$ $\mu \mathrm{F} / \mathrm{cm}^{2}$ and $G_{\text {soma }}=0.038 \mathrm{mS} / \mathrm{cm}^{2}$ for the capacitance and the conductance of the somatic membrane and $G=0.043 \mathrm{mS} / \mathrm{cm}^{2}$ and $R=469 \Omega \mathrm{cm}$ for the membrane conductance and the cytoplasmic resistivity of the neurites. We repeated the measurement and fit for two other neurons and found values of 0.9 $\mu \mathrm{F} / \mathrm{cm}^{2}$ and $0.8 \mu \mathrm{F} / \mathrm{cm}^{2}$ for $C_{\text {soma }}, 0.005 \mathrm{mS} / \mathrm{cm}^{2}$ and 0.016 $\mathrm{mS} / \mathrm{cm}^{2}$ for $G_{\text {soma }}, 0.03 \mathrm{mS} / \mathrm{cm}^{2}$ and $0.033 \mathrm{mS} / \mathrm{cm}^{2}$ for $G$, and 476 and $236 \Omega \mathrm{cm}$ for $R$. With $\chi^{2} /(N D)<2$ for the best fit in all three neurons, the experimental and simulated transient shapes agreed rather well (Press et al. 1997).

The value of $G_{\text {soma }}$ used for the model neuron was the least well defined input to the fit and can only be a rough estimate of the actual somatic membrane conductance. For that reason, we tested whether other values of $G_{\text {soma }}$ would also lead to adequate fits with different results for the other parameters. In fact, varying $G_{\text {soma }}$ in the physiological range could lead to fits with similarly good agreement with the experimental data as the best fit reported above. This variation of $G_{\text {soma }}$ left the values for $C_{\text {soma }}$ and $R$ virtually unchanged but could result in a significantly changed value obtained for the neuritic membrane conductance $G$, indicating that there is a trade-off between $G_{\text {soma }}$ and $G$.

Averaged over all three experiments, the neurite parameters obtained from the best fits are $G=0.035 \mathrm{mS} / \mathrm{cm}^{2}$ and $R=$ $\Omega \mathrm{cm}$, and the average neurite diameter is $d=5 \mu \mathrm{m}$. With these values, the electrotonic length constant $\lambda=\sqrt{d /(4 R G)}$ of the neurites is in the range of $\lambda \approx 1,000 \mu \mathrm{m}$. Given the typical length of cultured Lymnaea neurites of several hundred micrometers, their electrotonic length $L=l / \lambda$ is small but cannot be neglected. For such relatively short electrotonic lengths, Eq. 18 shows that the dominant cable parameter of influence for the synaptic conductance estimate is the cytoplasmic resistivity $R$. Fortunately, $R$ is the most reliable parameter obtained from the optical recordings.

\section{Theory for remote synapses}

Figure 1, $A$ and $B$, shows the equivalent circuits for a pair of electrically coupled neurons without and with neurites between the cell bodies and an electrical synapse. In the isopotential model in Fig. $1 A$, the effective synaptic conductance $g_{\text {syn }}$ can be computed from somatic recordings in response to current injection according to Eq. 8. With cable theory we derived

$$
\frac{1}{\overline{\bar{g}}_{\mathrm{syn}}}=\frac{1}{g_{\mathrm{syn}}} \frac{1}{\cosh L_{\mathrm{pre}} \cosh L_{\mathrm{post}}}-\lambda_{\mathrm{pre}} r_{\mathrm{pre}} \tanh L_{\mathrm{pre}}-\lambda_{\mathrm{post}} r_{\text {post }} \tanh L_{\mathrm{post}}
$$

which expresses the true synaptic conductance $\overline{\bar{g}}_{\text {syn }}$ between the neurite terminals in terms of $g_{\text {syn }}$ and the experimentally determined cable properties of the presynaptic and postsynaptic neurites. These are the resistances per unit length $r_{\text {pre }}$ and $r_{\text {post }}$, the geometric lengths $l_{\text {pre }}$ and $l_{\text {post }}$, the electrotonic length constants $\lambda_{\text {pre }}$ and $\lambda_{\text {post }}$, and the electrotonic lengths $L_{\text {pre }}=$ $l_{\text {pre }} / \lambda_{\text {pre }}$ and $L_{\text {post }}=l_{\text {post }} / \lambda_{\text {post }}$. Relation 18 is the approximation of Eq. 17 for electrotonically short neurites with $L_{\text {pre }} \ll 1$ and $L_{\text {post }} \ll 1$

$$
\frac{1}{\overline{\bar{g}}_{\text {syn }}}=\frac{1}{g_{\text {syn }}}-r_{\text {pre }} l_{\text {pre }}-r_{\text {post }} l_{\text {post }}
$$

The synaptic resistance $1 / \overline{\bar{g}}_{\text {syn }}$ is smaller than the apparent resistance $1 / g_{\text {syn }}$ because $1 / g_{\text {syn }}$ includes the axial resistances $r_{\text {pre }} l_{\text {pre }}$ and $r_{\text {post }} l_{\text {post }}$ of the two neurites.

\section{Conductances of remote synapses}

We used the average values of $G=0.035 \mathrm{mS} / \mathrm{cm}^{2}$ and $R=$ $394 \Omega \mathrm{cm}$ and the neurite lengths and diameters measured from photomicrographs of the coupled neuron pairs to compute with 
A

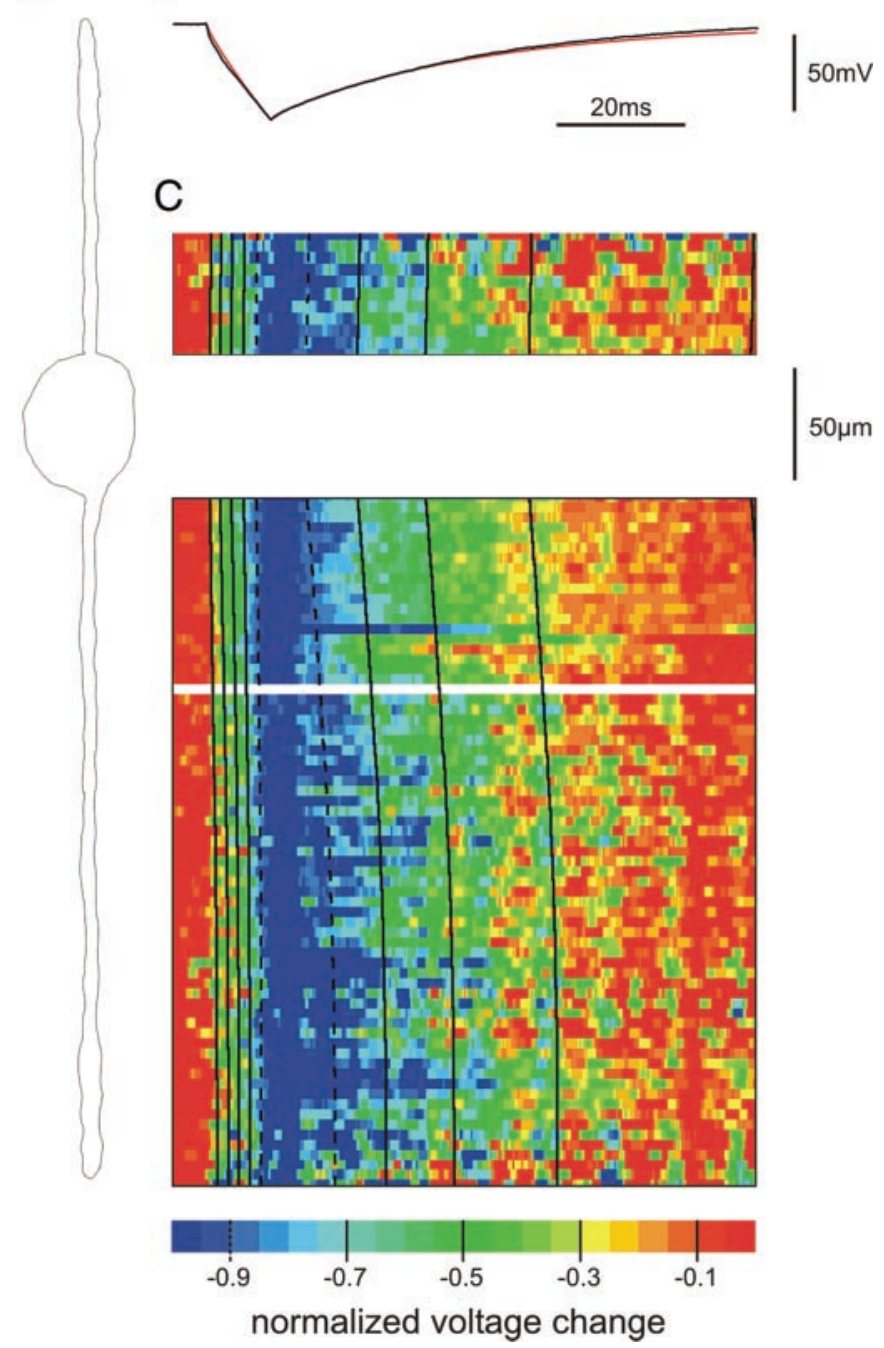

FIG. 4. Cable properties of cultured Lymnaea neurites. A: outline drawing of a snail neuron cultured on a linear pattern. The cell was stained with a voltage-sensitive dye and impaled with a microelectrode. $B$ : somatic voltage transient (black) of the neuron in response to a 10-ms hyperpolarizing current pulse of $-2 \mathrm{nA}$ injected into the cell body and simulated response (red) of the corresponding model neuron to the same current injection for a best fit of the electrical parameters. $C$ : color coded spatiotemporal map of the normalized fluorescence transients in the neurites and contour map of normalized simulated voltage transient for a best fit of the electrical parameters. The color code ranges from -1 (blue) to 0 (red) and the contour plot has solid lines at -0.1 , $-0.3,-0.5$, and -0.7 , and a dashed line at -0.9 . The hyperpolarization propagates fast throughout the neuron within a few milliseconds.

$E q .17$ the actual junctional conductance $\overline{\bar{g}}_{\text {syn }}$ from the estimated value $g_{\text {syn }}$ obtained with the isopotential model as described in the preceding text. The resulting values of $\overline{\bar{g}}_{\text {syn }}$ are shown in Fig. $5 A$ in a cumulative distribution function and fall between 0.1 and $1.2 \mathrm{nS}$.

For a more direct comparison, we plotted the synapse conductances $\overline{\bar{g}}_{\text {syn }}$ obtained with cable theory versus the conductances $g_{\text {syn }}$ computed with the isopotential theory in Fig. $5 B$. The isopotential theory obviously underestimates the synaptic conductance with $\overline{\bar{g}}_{\text {syn }}$, although the difference is small. The actual synapse conductances of the 11 cell pairs used here are on average $13 \%$ larger than would be expected from the isopotential model.

\section{I S C U S S I O N}

Our theoretical and experimental results indicate that neuritic cables between the (typically somatic) recording sites and the electrical synapse between two neurons can distort the conductance estimate for that synapse. This is particularly relevant in cases where electrical synapses are located far from the cell bodies and yet significantly shape the signaling between the coupled cells. Examples for this can be found in the Mauthner system of the goldfish and in rat hippocampus: In the goldfish, electrical synapses between the club endings of auditory afferent fibers and the distal part of the Mauthner cell's dendrite synchronize active fibers and promote the recruitment of new fibers via back-firing (Pereda et al. 1995). And in rat hippocampal slices, axo-axonal gap junctions allow for very fast signal transmission between principal neurons and are thought to enable highly precise signaling and high-frequency oscillations in networks of neurons (Schmitz et al. 2001).

\section{Coupling coefficient and $g_{\text {syn }}$}

A direct measure of the coupling by electrical synapses is the coupling coefficient $k_{\text {pre-post }}$, which is the ratio of the post- and presynaptic voltage change caused by a hyperpolarizing current injected into the presynaptic soma. For our geometrically simple cell pairs, the coupling coefficients mostly ranged between 0.02 and 0.12 (Fig. 3A), which puts them near the lower limit of the coefficients reported for neurons from different molluscan species both in vivo and in vitro (Benjamin and Pilkington 1986, Bodmer et al. 1984, Hadley et al. 1985, Magoski and Bulloch 1998, Magoski et al. 1994). Our cell pairs were grown on guiding substrates and formed synapses restricted to a single point of physical contact, whereas pairs grown under identical culture conditions on homogeneous substrates routinely established several points of physical contact and had about fourfold stronger synapses (Prinz and Fromherz 2000). So the small coupling coefficients reported here are most likely due to the restricted number of contacts between the two cells. Using the isopotential theory, we evaluated the synaptic conductances $g_{\text {syn }}$. With $g_{\text {syn }}<1 \mathrm{nS}$, we found them to be rather low compared with the values of typically several tens of nanoSiemens reported for molluscan nerve cells in vitro (Bodmer et al. 1988; Carrow and Levitan 1989) and in situ (Benjamin and Pilkington 1986; Bodmer et al. 1988; Kiss
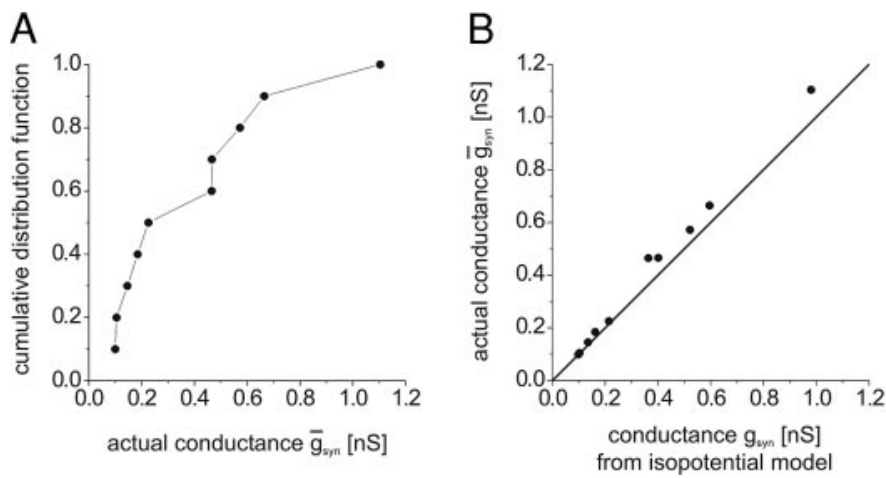

FIG. 5. Synaptic conductances. A: cumulative distribution function of synapse conductances $\overline{\bar{g}}_{\text {syn }}$ computed for 11 cultured snail cell pairs evaluated with the cable theory. $B$ : synapse conductances $\overline{\bar{g}}_{\text {syn }}$ computed with the cable theory plotted vs. $g_{\text {syn }}$ with the isopotential theory the same cells. All data are above the diagonal marking $\overline{\bar{g}}_{\text {syn }}=g_{\text {syn }}$. 
1979; Wildering and Janse 1992). Although the values computed for $k_{\text {pre-post }}$ and $G_{\text {syn }}$ both indicate weak synaptic coupling, the correlation between the two measures is weak (Fig. $3 C$ ), presumably due to the variation of the input conductance of the neurons.

\section{Cable properties from optical imaging}

Cable properties of neurons in a number of different systems have been computed from somatic voltage transients in response to current injections at the cell body (Coleman and Miller 1989; Fleshman et al. 1988; Jackson 1992; Stafstrom et al. 1984). In cells whose somatic membrane properties differ from those of the neurites (Durand 1984, Kawato 1984), this parameter identification problem has, however, been demonstrated to be ill-posed in that very small errors in measured data can lead to large and unpredictable errors in parameter estimates (White et al. 1992). To avoid that problem, we took advantage of the simple geometry and good optical accessibility of cultured Lymnaea nerve cells and determined their cable properties from voltage-sensitive dye measurements of voltage transients in the entire cell rather than at the cell body alone (Fromherz and Müller 1994). We estimated the electrical parameters by fitting voltage transients simulated with a passive multi-compartment model to voltage transients reconstructed from fluorescence measurements throughout the cell and transients recorded directly at the cell body (Fig. 4). The propagation of voltage changes along the neurites was very fast, resulting in rather feature-less optical maps (Fig. 4C). For that reason, the evaluation of the cable parameters was less reliable than with neurites where larger propagation delays in the distal parts were observed (Meyer et al. 1997).

We found an average cytoplasmic resistivity $R$ of about 400 $\Omega \mathrm{cm}$, a value far higher than the classical $35 \Omega \mathrm{cm}$ for the squid giant axon (Hodgkin and Huxley 1952) but similar to values reported for other narrower neurites such as the $250 \Omega \mathrm{cm}$ assigned to cultured leech neurons (Fromherz and Müller 1994; Fromherz and Vetter 1992) and the 340 and $300 \Omega \mathrm{cm}$ found for rat hippocampal neurons (Major et al. 1994; Meyer et al. 1997). The average neuritic membrane conductance $G=0.035$ $\mathrm{mS} / \mathrm{cm}^{2}$ that led to the best fits in three experiments is similar to values found with imaging techniques in cultured leech neurons $\left(G=0.05 \mathrm{mS} / \mathrm{cm}^{2}\right)$ (Fromherz and Müller 1994) and cultured rat hippocampal pyramidal neurons $(G=0.07 \mathrm{mS} /$ $\mathrm{cm}^{2}$ ) (Meyer et al. 1997). The high propagation velocity evident in Fig. $4 C$ is therefore largely due to the unusually large neurite diameters of Lymnaea neurites cultured on patterned substrates.

\section{Impact of neuritic cables on synaptic conductance estimates}

We consider Eq. 17 to be the main result of this paper. It relates the junctional conductance of a remote electrical synapse to experimentally accessible currents and voltages and neuritic parameters and will allow researchers to obtain more refined estimates of electrical synapse strength that they could previously achieve by applying the isopotential cell pair model of Bennett (1966). To illustrate the extent to which neuritic cables between the cell bodies and remote electrical synapses can influence the synaptic conductance estimate, the relationship between the apparent synaptic conductance $g_{\text {syn }}$ obtained with Bennett's model and the actual junctional conductance $\overline{\bar{g}}_{\text {syn }}$ is illustrated in Fig. 6, $A$ and $B$, for two sets of cable parameters. In Fig. 6A, the parameters were $\lambda_{\text {pre }}=\lambda_{\text {post }}=$ $1,083 \mu \mathrm{m}, r_{\text {pre }}=r_{\text {post }}=1.3 \times 10^{9} \Omega / \mathrm{cm}$, and $l_{\text {pre }}=l_{\text {post }}=$ $30-1,000 \mu \mathrm{m}$. These values for the length constant $\lambda$ and the resistance per unit length $r$ follow from $d_{\text {pre }}=d_{\text {post }}=6 \mu \mathrm{m}$, $R_{\text {pre }}=R_{\text {post }}=394 \Omega \mathrm{cm}$, and $G_{\text {pre }}=G_{\text {post }}=0.035 \mathrm{mS} / \mathrm{cm}^{2}$, which are the values we obtained for snail neurites cultured on linear lanes (see RESULTS). The figure shows that the discrepancy between $\overline{\bar{g}}_{\text {syn }}$ and $g_{\text {syn }}$ increases for long neurites and large synaptic conductances. For $\overline{\bar{g}}_{\mathrm{syn}} \approx 0.5 \mathrm{nS}$ and $l \approx 300 \mu \mathrm{m}$, typical for the neurons used here, the devations are, however, modest.

In Fig. $6 B$, we chose $\lambda_{\text {pre }}=\lambda_{\text {post }}=354 \mu \mathrm{m}, r_{\text {pre }}=r_{\text {post }}=$ $25 \times 10^{9} \Omega / \mathrm{cm}$, and $l_{\text {pre }}=l_{\text {post }}=30-1,000 \mu \mathrm{m}$. These values for $\lambda$ and $r$ follow from $d_{\text {pre }}=d_{\text {post }}=1 \mu \mathrm{m}, R_{\text {pre }}=$ $R_{\text {post }}=200 \Omega \mathrm{cm}$, and $G_{\text {pre }}=G_{\text {post }}=0.1 \mathrm{mS} / \mathrm{cm}^{2}$, which are typical values for various mammalian neurons (Clements and Redman 1989; Koch 1999; Meyer et al. 1997; Stafstrom et al. 1984). Here, significant discrepancies between $\overline{\bar{g}}_{\text {syn }}$ and $g_{\text {syn }}$ appear for much shorter neurites and much smaller synaptic conductances.

Figure 6 and Eq. 17 indicate that the rather modest differences of only about $13 \%$ between the actual junctional conductance $g_{\text {syn }}$ between snail neurite tips and the estimate $g_{\text {syn }}$ obtained with the isopotential model are due to the small electrotonic lengths and weak electrical synapses of the cultured pairs of snail neurons- both are factors that favor good agreement between the isopotential and the nonisopotential model. For less compact and more strongly coupled pairs of neurons, the error in the synaptic conductance estimate due to the assumption of isopotential cells will, however, be much more dramatic.

\section{Applicability of the nonisopotential theory in vivo}

In electrically coupled cell pairs in vivo, the effect of the neuritic cable on the discrepancy of $g_{\text {syn }}$ and $\overline{\bar{g}}_{\text {syn }}$ is likely to be more severe than in cultured snail neurons. The synapse conductance estimates $g_{\text {syn }}$ can be as low as a few percent of the actual value $\overline{\bar{g}}_{\text {syn }}$ if the synapse is strong and far away from the
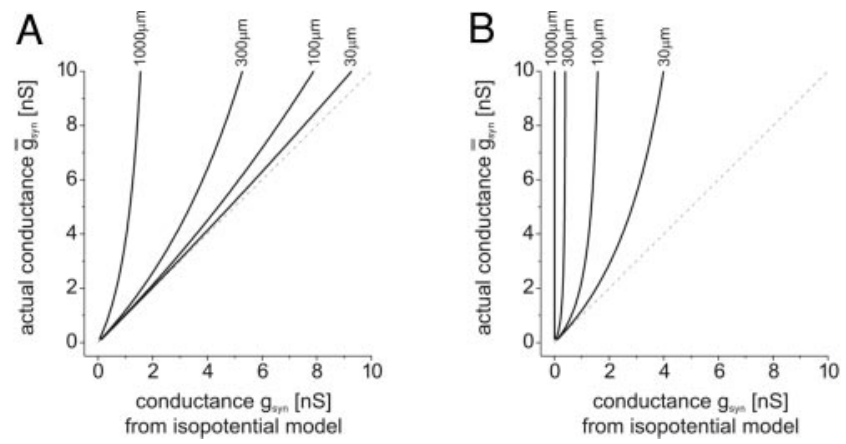

FIG. 6. Effect of cable properties on synaptic conductance estimates. A: synapse conductances $\overline{\bar{g}}_{\text {syn }}$ between the terminals of 2 neurites plotted vs. $g_{\text {syn }}$ computed under the assumption of isopotential cells. The 4 curves show the values computed for neurite lengths $l_{\text {pre }}=l_{\text {post }}$ of $30,100,300$, and $1,000 \mu \mathrm{m}$ using the cable parameters of the cultured Lymnaea neurites. B: same as in $A$ but using different cable parameters: the specific membrane conductance in this case was $G=0.1 \mathrm{mS} / \mathrm{cm}^{3}$, the cytoplasmic resistivity was $R=200 \Omega$, and the neurite diameter was $d=1 \mu \mathrm{m}$, as reported for various mammalian neurons. 
cell bodies. Of course, to use Eq. 17 for a determination of the junctional conductance from somatic voltage recordings, one needs to know the length $l$ of the neurites that connect the two cell bodies to the synapse, their resistance $r$ per unit length, and their electrotonic length constant $\lambda$. For coupled cell pairs in vivo, only rough estimates of these values may be available.

The applicability of the model shown in Fig. $1 B$ to electrically coupled neurons with extensive neuritic trees is further compromised by the fact that multiple coupling sites at different electrotonic distances from the cell body might exist and that neurites can extend beyond the coupling site. However, simple cable models have been successfully applied to complex neuritic trees before (Jackson 1992; Rose and Dagum 1988; Stafstrom et al. 1984; Whitehead and Rosenberg 1993), and even the isopotential cell pair model (Fig. 1A) has been used for obviously nonisopotential pairs of neurons (Benjamin and Pilkington 1986; Bodmer et al. 1988; Kiss 1979). We believe that in such situations the application of relation ( $E q$. 17) can lead to better estimates of the junctional conductances of electrical synapses than could previously be obtained by assuming isopotentiality, and can do so without the need for detailed compartmental models of the neurons involved.

\section{A P P E N D IX}

To allow a comparison of the current balances (Eqs. 9 and 10) of the nonisopotential model with the current balances (Eqs. 2 and 3) of the isopotential model, we eliminate the currents $i_{\text {pre,prox }}^{\text {memb }}$ and $i_{\text {post,prox }}^{\text {memb }}$ through the membrane of the proximal pre- and postsynaptic neurites and the voltages $V_{\text {pre }}^{\text {tip }}$ and $V_{\text {post }}^{\text {tip }}$ at the tips of the pre- and postsynaptic neurites with the help of cable theory. According to Rall (1959), the currents through the membranes of the proximal neurites can be expressed by Eqs. $A 1$ and $A 2$, where $V_{\text {pre }}$ and $V_{\text {post }}$ are the voltages at the cell bodies

$$
\begin{gathered}
i_{\text {preprox }}^{\text {memb }}=\left(V_{\text {pre }}-\overline{\bar{V}}_{\text {pre,rest }}\right) \frac{\tanh L_{\text {pre }}}{\lambda_{\text {pre }} r_{\text {pre }}}+i_{\text {pre,tip }}^{\text {axial }} \frac{1-\cosh L_{\text {pre }}}{\cosh L_{\text {pre }}} \\
i_{\text {post.prox }}^{\text {memb }}=\left(V_{\text {post }}-\overline{\bar{V}}_{\text {post, rest }}\right) \frac{\tanh L_{\text {post }}}{\lambda_{\text {post }} r_{\text {post }}}+i_{\text {post,tip }}^{\text {axial }} \frac{1-\cosh L_{\text {post }}}{\cosh L_{\text {post }}}
\end{gathered}
$$

The axial currents $i_{\text {pre,tip }}^{\text {axial }}$ and $i_{\text {post,tip }}^{\text {axial }}$ flowing along the proximal neurites at their tips are equal to the current flowing through the synapse, with a minus sign for $i_{\text {post,tip }}^{\text {axial }}$ because the axial current is defined as flowing away from the soma

$$
i_{\text {pret,ip }}^{\text {axial }}=\overline{\bar{g}}_{\text {syn }}\left(V_{\text {pre }}^{\text {tip }}-V_{\text {post }}^{\text {tip }}\right)=-i_{\text {post,tip }}^{\text {axial }}
$$

With this and Eqs. $A 1$ and $A 2$, the steady-state current balances Eqs. 9 and 10 simplify to Eqs. $A 4$ and $A 5$, where $\overline{\bar{g}}_{\text {pre }}$ and $\overline{\bar{g}}_{\text {post }}$ are the input conductances of the two neurons as defined by $E q .13$

$$
\begin{aligned}
& \overline{\bar{g}}_{\text {pre }}\left(V_{\text {pre }}-\overline{\bar{V}}_{\text {pre,rest }}\right)+\overline{\bar{g}}_{\text {syn }}\left(V_{\text {pre }}^{\mathrm{tip}}-V_{\text {post }}^{\mathrm{tip}}\right) \frac{1}{\cosh L_{\text {pre }}}=i_{\text {inj }} \\
& \overline{\bar{g}}_{\text {post }}\left(V_{\text {post }}-\overline{\bar{V}}_{\text {post,rest }}\right)+\overline{\bar{g}}_{\text {syn }}\left(V_{\text {post }}^{\mathrm{tip}}-V_{\text {pre }}^{\mathrm{tip}}\right) \frac{1}{\cosh L_{\text {post }}}=0
\end{aligned}
$$

Equations $A 4$ and $A 5$ still contain the potential difference $V_{\text {pre }}^{\text {tip }}-$ $V_{\text {post }}^{\text {tip }}$ between the tips of the two neurites. We use the relations $E q s$. $A 6$ and $A 7$ from cable theory (Rall 1959) to express it in terms of the somatic potentials and properties of the neurons and synapse

$$
V_{\text {pre }}^{\text {tip }}-\overline{\bar{V}}_{\text {pre,rest }}=\frac{1}{\cosh L_{\text {pre }}}\left(V_{\text {pre }}-\overline{\bar{V}}_{\text {pre,rest }}\right)-\lambda_{\text {pre }} r_{\text {pre }} \tanh L_{\text {pre }} i_{\text {pre,tip }}^{\text {axial }}
$$

$$
V_{\text {post }}^{\text {tip }}-\overline{\bar{V}}_{\text {post,rest }}=\frac{1}{\cosh L_{\text {post }}}\left(V_{\text {post }}-\overline{\bar{V}}_{\text {post,rest }}\right)-\lambda_{\text {post }} r_{\text {post }} \tanh L_{\text {post }} t_{\text {post,tip }}^{\text {axial }}
$$

Using Eq. $A 3$ in $A 6$ and $A 7$, subtracting them and solving for $V_{\text {pre }}^{\text {tip }}$ results in $E q$. A8, with the abbreviation $\lambda$ given by $E q .15$

$$
\begin{aligned}
V_{\text {pre }}^{\mathrm{tip}}-V_{\text {post }}^{\mathrm{tip}}=\frac{\gamma}{g_{\text {syn }}}[ & \left(V_{\text {pre }}-\overline{\bar{V}}_{\text {pre,rest }}\right) \cosh L_{\text {post }}-\left(V_{\text {post }}-\overline{\bar{V}}_{\text {post,rest }}\right) \\
& \left.\times \cosh L_{\text {pre }}+\left(\overline{\bar{V}}_{\text {pre,rest }}-\overline{\bar{V}}_{\text {post,rest }}\right) \cosh L_{\text {pre }} \cosh L_{\text {post }}\right]
\end{aligned}
$$

If this is used in Eqs. A4 and A5, the current balances for the nonisopotential model can be brought into a form homologous to the balances (Eqs. 2 and 3) in the isopotential model. The resulting Eqs. 11 and 12 allow a direct comparison of homologous parameters in the two models.

The project was supported by a generous grant of the Bundesministerium für Bildung und Forschung.

Present address of A. A. Prinz: Volen Center for Complex Systems, Brandeis University, Waltham, MA 02454.

\section{REFERENCES}

Benjamin PR and Pilkington JB. The electrotonic location of low-resistance intercellular junctions between a pair of giant neurons in the snail Lymnaea. J Physiol 370: 111-126, 1986.

Bennett MVL. Physiology of electrotonic junctions. Ann NY Acad Sci 137: 509-539, 1966

Bennett MVL. Gap junctions as electrical synapses. J Neurocytol 26: 349366, 1997.

Bodmer R, Dagan D, and Levitan IB. Chemical and electrotonic connections between Aplysia neurons in primary culture. J Neurosci 4: 228-233, 1984.

Bodmer R, Verselis V, Levitan IB, and Spray DC. Electrotonic synapses between Aplysia neurons in situ and in culture: aspects of regulation and measurements of permeability. J Neurosci 8: 1656-1670, 1988

Carrow GM and Levitan IB. Selective formation and modulation of electrical synapses between cultured Aplysia neurons. J Neurosci 9: 3657-3664, 1989.

Clements JD and Redman SJ. Cable properties of cat spinal motoneurons measured by combining voltage clamp, current clamp and intracellular staining. J Physiol 409: 63-87, 1989.

Coleman PA and Miller RF. Measurement of passive membrane parameters with whole cell recording from neurons in the intact amphibian retina. J Neurophysiol 61: 218-230, 1989.

Durand D. The somatic shunt cable model for neurons. Biophys $J$ 46: 645653, 1984.

Edwards DH, Yeh SR, and Krasne FB. Neuronal coincidence detection by voltage-sensitive electrical synapses. Proc Natl Acad Sci USA 95: 71457150, 1998.

Ephardt $\mathbf{H}$ and Fromherz P. Fluorescence of amphiphilic hemicyanine dyes without free double bonds. J Phys Chem 97: 4540-4547, 1993.

Fleshman JW, Segev I, and Burke RE. Electrotonic architecture of typeidentified $\alpha$-motoneurons in the cat spinal cord. J Neurophysiol 60: 60-85, 1988.

Fromherz $\mathbf{P}$ and Müller CO. Voltage-sensitive fluorescence of amphiphilic hemicyanine dyes in neuron membrane. Biochim Biophys Acta 115: 111222, 1993.

Fromherz P and Müller CO. Cable properties of a straight neurite of a leech neuron probed by a voltage-sensitive dye. Proc Natl Acad Sci USA 91: 4604-4608, 1994.

Fromherz $\mathbf{P}$ and Schaden H. Defined neuronal arborizations by guided outgrowth of leech neurons in culture. Eur J Neurosci 6: 1500-1504, 1994.

Fromherz $\mathbf{P}$ and Vetter T. Cable properties of arborized Retzius cells of the leech in culture as probed by a voltage-sensitive dye. Proc Natl Acad Sci USA 89: 2041-2045, 1992.

Hadley RD, Bodnar DA, and Kater SB. Formation of electrical synapses between isolated, cultured Helisoma neurons requires mutual neurite elongation. J Neurosci 5: 3145-3153, 1985

Hodgkin AL and Huxley AF. A quantitative description of membrane current and its application to conduction and excitation in nerve. J Physiol 117: 500-544, 1952. 
Jackson MB. Cable analysis with the whole cell patch clamp. Biophys $J$ 61: 756-766, 1992.

Kawato M. Cable properties of a neuron model with non-uniform membrane resistivity. J theor Biol 111: 149-169, 1984

Kiehn $\mathbf{O}$ and Tresch M. Gap junctions and motor behaviour. Trends Neurosci 25: $108-115,2002$.

Kiss I. Electrotonic transmission between giant neurons identified in the CNS of Lymnaea stagnalis. Acta Biol Acad Sci Hung 30: 103-119, 1979.

Koch C. Appendix A. In: Biophysics of Computation. New York: Oxford, 1999, p. 481-482.

Magoski NS and Bulloch AGM. Trophic and contact conditions modulate synapse formation between identified neurons. J Neurophysiol 79: 3279 3283, 1998.

Magoski NS, Syed NI, and Bulloch AGM. A neuronal network from the mollusc Lymnaea stagnalis. Brain Res 645: 201-214, 1994.

Major G, Larkman AU, Jonas P, Sakmann B, and Jack JJB. Detailed passive cable models of whole-cell recorded CA3 pyramidal neurons in rat hippocampal slices. J Neurosci 14: 4613-4638, 1994.

Manor Y, Rinzel J, Segev I, and Yarom Y. Low-amplitude oscillations in the inferior olive: a model based on electrical coupling of neurons with heterogenous channel densities. J Neurophysiol 77: 2736-2752, 1997.

Marder E. Electrical synapses: Beyond speed and synchrony to computation. Curr Biol 8: R795-R797, 1998.

Meyer E, Müller CO, and Fromherz P. Cable properties of dendrites in hippocampal neurons of the rat mapped by a voltage-sensitive dye. Eur J Neurosci 9: 778-785, 1997.

Müller CO. Spannungsprofile Linearer Neurite: Messungen mit Fluoreszenzfarbstoffen an Neuronen von Hirudo Medicinalis (PhD thesis). Ulm, Germany: University Ulm, 1994.

Pereda AE, Bell TD, and Faber DS. Retrograde synaptic communication via gap junctions coupling auditory afferents to the Mauthner cell. J Neurosci 15: 5943-5955, 1995.

Press WH, Teukolsky SA, Vetterling WT, and Flannery BP. Numerical Recipes in C (2nd ed.). Cambridge, UK: Cambridge Univ. Press, 1997.

Prinz AA. Towards Defined Networks: Guided Growth, cable Transmission and Electrical Synapses of Cultured Snail Neurons (PhD thesis). Munich, Germany: Technical University Munich, 2000.
Prinz AA and Fromherz P. Electrical synapses by guided growth of cultured neurons from the snail Lymnaea stagnalis. Biol Cybern 82: L1-L5, 2000.

Rall W. Branching dendritic trees and motoneuron membrane resistivity. Exp Neurol 1: 491-527, 1959.

Rall W. Membrane potential transients and membrane time constant of motoneurons. Exp Neurol 2: 503-532, 1960.

Ridgway RL, Syed NI, Lukowiak K, and Bulloch AGM. Nerve growth factor (NGF) induces sprouting of specific neurons of the snail, Lymnaea stagnalis. J Neurobiol 22: 377-390, 1991.

Rose PK and Dagum A. Nonequivalent cylinder models of neurons: interpretation of voltage transients generated by somatic current injection. $\mathrm{J} \mathrm{Neu}$ rophysiol 60: 125-148, 1988.

Schmitz D, Schuchmann S, Fisahn A, Draguhn A, Buhl EH, PetraschParwez E, Dermietzel R, Heinemann U, and Traub RD. Axo-axonal coupling: a novel mechanism for ultrafast neuronal communication. Neuron 31: 831-840, 2001.

Sherman A and Rinzel J. Rhythmogenic effects of weak electrotonic coupling in neuronal models. Proc Natl Acad Sci USA 89: 2471-2474, 1992.

Stafstrom CE, Schwindt PC, and Crill WE. Cable properties of Layer V neurons from cat sensorimotor cortex in vitro. J Neurophysiol 52: 278-289, 1984

Traub RD, Kopell N, Bibbig A, Buhl EH, LeBeau FEN, and Whittington MA. Gap junctions between interneuron dendrites can enhance synchrony of gamma oscillations in distributed networks. J Neurosci 21: 9478-9486, 2001

White JA, Manis PB, and Young ED. The parameter identification problem for the somatic shunt cable model. Biol Cybern 66: 307-318, 1992.

Whitehead RR and Rosenberg JR. On trees as equivalent cables. Proc $R$ Soc Lond B Biol Sci 252: 103-108, 1993.

Wildering WC and Janse C. Serotonergic modulation of junctional conductance in an identified pair of neurons in the mollusk Lymnaea stagnalis. Brain Res 595: 343-352, 1992.

Wong RG, Martel EC, and Kater SB. Conditioning factor(s) produced by several molluscan species promote neurite outgrowth in cell culture. $J$ Exp Biol 105: 389-393, 1983. 